\title{
INTEGRATED ARCHITECTURE OF ACTUATOR FAULT DIAGNOSIS AND ACCOMMODATION
}

\author{
Rim Hamdaoui, Safa Guesmi, Rafika Elharabi and Med Naceur Abdelkrim \\ UR. Modelling, Analysis and Systems Control, University of Gabes, Gabes, Tunisia \\ rim.hamdaoui@gmail.com, guesmi_safa@hotmail.fr, rafikaharabi@yahoo.fr, \\ naceur.abdelkrim@enig.rnu.tn
}

\begin{abstract}
This paper deals with the design of an integrated scheme of actuator fault diagnosis and accommodation. The fault detection, isolation and estimation are given by the diagnosis task based on an Unknown Input Observer UIO. The UIO allows a robust diagnosis throughout decoupling disturbances from faults and provides estimation for the faults amplitude. This latter is used in order to recompute, on line, a feedback control gain that guaranteeing the fault accommodation and the compensation of its effect. The control gain is determinate based on the Pseudo-Inverse Method PIM.
\end{abstract}

\section{KEYWORDS}

Integrated design, Robust Diagnosis, Fault accommodation, UIO, PIM \& Actuator fault

\section{INTRODUCTION}

A great attention has been paid to the problem of fault detection and diagnosis and active fault tolerant control systems. This is a consequence of the continued progress of the industrial process that increases the demand for high reliability and safety.

Then, several approaches have been developed in the field of fault diagnosis and fault tolerance. Model-based methods of fault detection were developed by using input and output signals and applying dynamic process models ([1], [2], [3]...). These methods are based on parameter estimation, parity equations or state observers and aim to detect the fault, isolate the faulty component or sub-system and identify the fault's range.

Research in Active Fault Tolerant Controllers AFTC area has been, also, gaining increasing consideration (a bibliographical review is presented in [2]). AFTC are synthesized for the purpose of accommodating the system's operation with the fault and maintaining an acceptable level of performances during the degraded mode.

Naturally, the accommodation task uses the information about the faults that are supplied from the Fault Detection and Diagnosis task. However, the majority of AFTC methods assume that the FDD task is perfect and provides precise information about the post-fault system behaviour, which is not, really, the case.

In practise situations, it is important to cope with FDD uncertainty and delays while developing an AFTC design. Indeed, the FDD performances influence directly the quality of the fault accommodation or the system reconfiguration. Thus, viewing the FDD and the AFTC as a whole integrated architecture brings a significant supply in the domain of AFTC systems. Some 
researchers were interested by the design of integrated FDD and FTC methodologies in order to improve the control performance using the fault information generated by the diagnosis module, for instance in the works [4] and [5].

The present integrated design is constituted by a FDD block based on an Unknown Input Observer that is used for residual generation, fault detection and estimation. The estimate of the fault is then used to recalculate, on line, the control gain and applying the accommodating control, based on the Pseudo-Inverse Method PIM, which constitutes the second module of the integrated architecture.

The second section of this work states the problem by presenting the nominal and the faulty modes of the system. The third section proposes the integrated design of FDD and AFTC by developing an UIO for the fault detection and estimation and explaining the use of the FDD information for the design of the accommodating control.

The fourth section illustrates the found results by applying the methodology on a VTOL aircraft. Finally, concluding remarks and prospects are given in the fifth section.

\section{Problem statement}

Consider a nominal LTI system represented by:

$S_{\text {nom }}:\left\{\begin{array}{l}\dot{x}(t)=A x(t)+B u(t) \\ y(t)=C x(t)\end{array}\right.$

$x \in R^{n}$ is the state vector, $y \in R^{m}$ is the measure vector and $u \in R^{p}$ is the control vector. $A$ and $B$ are constant matrices with suitable dimensions.

The control objective, in nominal mode, is allowing to the system in closed loop, a perfect follow to the nominal model given by the equation (2) within a mission finite time $t_{m i s}$ :

$\dot{x}(t)=M x(t)+N e(t)$

The matrices $M$ and $N$ are given and $M$ is chosen to be stable. $e(t) \in R^{q}$ is an arbitrary input vector. The nominal control law, guaranteeing the system's stability, is expressed by:

$u(t)=G_{n} e(t)-K_{n} x(t)$

The matrices $K_{n}$ and $G_{n}$ are computed through the resolution of the following equalities:

$\left\{\begin{array}{l}A-B K_{n}=M \\ B G_{n}=N\end{array}\right.$

Assuming that $B$ is of full rank column, so that $\operatorname{rank}(B)=p$, the unique solution of equation (4) is expressed by [6]:

$\left\{\begin{array}{l}K_{n}=B^{+}(A-M) \\ G_{n}=B^{+} N\end{array}\right.$

where $B^{+}$is the left pseudo-inverse of $B$ verifying $B^{+} B=I_{p}$ with $I_{p}$ is the identity matrix. 
In view of the occurrence of an actuator fault at an instant $t_{f}$, the system is, therefore, represented by:

$S_{\text {def }}:\left\{\begin{array}{l}\dot{x}(t)=A x(t)+B(u(t)+f(t)) \\ y(t)=C x(t)\end{array}\right.$

Considering $\gamma_{i}, i=1, \ldots, p$, that indicates the $i^{\text {th }}$ actuator state [7], $f(t)$ is resulted from a loss of control factor efficiency when $0<\gamma_{i} \leq 1$. The actuator fault's model is given by the equation (7):

$f(t)=\left(I_{p}-\Gamma\right) u(t), \quad \Gamma=\operatorname{diag}\left(\gamma_{1}, \gamma_{2}, \cdots, \gamma_{p}\right)$

In order to compensate the fault's effect on the process response and performances, immediate information about the fault is needed on line. The FDD procedure is charged to precise the occurrence's instant, the location and the magnitude of the fault. Then, the active fault tolerant control system exploits this information to elaborate an accommodating control law.

The following section gives details about the FDD and the FTC modules within an integrated schema as shown in Figure 1.

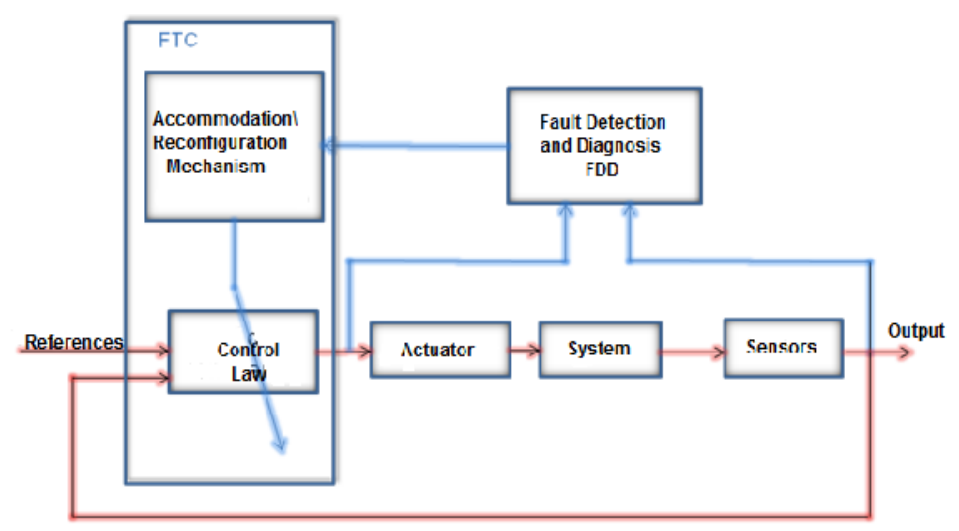

Figure 1. Integrated design of FDD and FTC.

\section{INTEGRATED ARCHITECTURE OF FDD AND FTC}

\subsection{Fault Detection and Diagnosis FDD}

The fault detection and diagnosis task aims to generate residual signals and evaluate them to decide about the occurrence or not of a fault, to isolate the fault and to identify it. In this work, we deal with the FDD based on Unknown Input Observer UIO.

\subsubsection{Unknown Input Observer design}

In the presence of both actuator fault and unknown disturbance $d \in \mathfrak{R}^{l}$, the system (6) becomes: 
$\left\{\begin{array}{l}\dot{x}(t)=A x(t)+B(u(t)+f(t))+D d(t) \\ y(t)=C x(t)\end{array}\right.$

We assume that $\operatorname{rank}(C)=m, \operatorname{rank}(D)=l, \operatorname{rank}(f)=p, \operatorname{rank}(C D)=l, \operatorname{rank}(C F)=p$, $(m \geq p),(m \geq 1+p)$ and $(m \geq 1)$. In order to elaborate a full-order observer, we consider the non singular transformation matrix [8]:

$T=\left[\begin{array}{lll}N & D & B\end{array}\right], \quad N \in \Re^{n \times(n-l-p)}$

$N$ is an arbitrary matrix chosen so that $T$ is non singular. Then, the system given by (8) can be rewritten under the following form:

$\left\{\begin{array}{l}\bar{x}(t)=\bar{A} \bar{x}(t)+\bar{B}(u(t)+f(t))+\bar{D} d(t) \\ \bar{y}(t)=\bar{C} \bar{x}(t)\end{array}\right.$

where:

$$
\begin{gathered}
x=T \bar{x}=T\left[\begin{array}{l}
\bar{x}_{1} \\
\overline{x_{2}} \\
\overline{x_{3}}
\end{array}\right] \quad \bar{A}=T^{-1} A T=\left[\begin{array}{ccc}
\overline{A_{11}} & \overline{A_{12}} & \overline{A_{13}} \\
\overline{A_{21}} & \overline{A_{22}} & \overline{A_{23}} \\
\overline{A_{31}} & \overline{A_{32}} & \overline{A_{33}}
\end{array}\right] \quad \bar{B}=T^{-1} B=\left[\begin{array}{c}
\bar{B}_{1} \\
\bar{B}_{2} \\
\overline{B_{3}}
\end{array}\right]=\left[\begin{array}{c}
0 \\
0 \\
I_{p}
\end{array}\right] \\
\bar{D}=T^{-1} D=\left[\begin{array}{c}
0 \\
I_{l} \\
0
\end{array}\right] \quad \bar{C}=C T=\left[\begin{array}{lll}
C N & C
\end{array}\right]
\end{gathered}
$$

with $\bar{x}_{1} \in \mathfrak{R}^{n-l-p}, \bar{x}_{2} \in \mathfrak{R}^{l}, \bar{x}_{3} \in \mathfrak{R}^{p}$.

The differential equations of the relation (10) corresponding to the state sub-vectors $\bar{x}_{2}$ and $\bar{x}_{3}$ directly engross the unknown input $d$ and the fault $f$. By dropping these differential equations, we obtain an unknown input and fault free system:

$$
\begin{aligned}
& {\left[\begin{array}{lll}
I_{n t p} & 0 & 0
\end{array}\right] \dot{x}(t)=\left[\begin{array}{lll}
\bar{A}_{1} & \bar{A}_{2} & \bar{A}_{3}
\end{array}\right] \bar{x}(t)+\bar{B} u(t)} \\
& y(t)=\left[\begin{array}{lll}
C N & C D & C B
\end{array}\right] \bar{x}(t)
\end{aligned}
$$

Consider a non-singular matrix $U$ described as:

$$
U=\left[\begin{array}{lll}
C D & C B & Q
\end{array}\right], \quad Q \in \Re^{m \times(m-l-p)}
$$

$Q$ is an arbitrary matrix chosen so that $U$ is non-singular. We define $U^{-1}$ as:

$U^{-1}=\left[\begin{array}{l}U_{1} \\ U_{2} \\ U_{3}\end{array}\right], \quad U_{1} \in \mathfrak{R}^{l \times m}, U_{2} \in \mathfrak{R}^{p \times m}, U_{3} \in \mathfrak{R}^{(m-l-p) \times m}$

Therefore, we obtain: 
International Journal of Instrumentation and Control Systems (IJICS) Vol.2, No.3, July 2012

$U^{-1} U=\left[\begin{array}{c}U_{1} \\ U_{2} \\ U_{3}\end{array}\right]\left[\begin{array}{lll}C D & C B & Q\end{array}\right]=\left[\begin{array}{ccc}U_{1} C D & U_{1} C B & U_{1} Q \\ U_{2} C D & U_{2} C B & U_{2} Q \\ U_{3} C D & U_{3} C B & U_{3} Q\end{array}\right]=\left[\begin{array}{ccc}I_{l} & 0 & 0 \\ 0 & I_{p} & 0 \\ 0 & 0 & I_{m-l-p}\end{array}\right]$

The multiplication of (12) by $U^{-1}$ gives:

$U_{1} y(t)=U_{1} C N \bar{x}_{1}(t)+\bar{x}_{2}(t)$

$U_{2} y(t)=U_{2} C N x_{1}(t)+\bar{x}_{3}(t)$

$U_{3} y(t)=U_{3} C N x_{1}(t)$

We substitute (16-a) and (16-b) in (12) and we combine it with (16-c), we get:

$\dot{\overline{x_{1}}}(t)=\tilde{A}_{1} \overline{x_{1}}+\overline{B_{1}} u(t)+E_{1} y(t)$

$\bar{y}(t)=\tilde{C}_{1} \bar{x}_{1}(t)$

with $\tilde{A}_{1}=\bar{A}_{11}-\bar{A}_{12} U_{1} C N-\bar{A}_{13} U_{2} C N, E_{1}=\bar{A}_{12} U_{1}+A_{13} U_{2}, \tilde{C}_{1}=U_{3} C N$ and $\bar{y}=U_{3} y$

Under the condition that the pair $\left(\tilde{A}_{1}, \tilde{C}_{1}\right)$ is observable (detectable), an unknown input observer can be elaborated to the system given by (17), expressed as:

$$
\begin{gathered}
\dot{w}(t)=\left(\tilde{A}_{1}-L \tilde{C}_{1}\right) w(t)+\bar{B}_{1} u(t)+L \bar{y}(t)+E_{1} y(t) \\
\quad=\left(\tilde{A}_{1}-L \tilde{C}_{1}\right) w(t)+\bar{B}_{1} u(t)+\left(L U_{3}+E_{1}\right) y(t)
\end{gathered}
$$

$w \in \mathfrak{R}^{n-l-p}$ is the estimation of $\bar{x}_{1}, L \in \mathfrak{R}^{(n-l-p) \times(m-l-p)}$ is a pre-specified observer gain matrix that makes the matrix $\tilde{A_{1}}-L \tilde{C_{1}}$ stable.

The state estimation error dynamics follows the equation:

$\dot{e}_{\bar{x}_{1}}(t)=w(t)-\bar{x}_{1}(t)=\left(\tilde{A}_{1}-L \tilde{C}_{1}\right) e_{\overline{\bar{x}}_{1}}(t)$

If $\tilde{A_{1}}-L \tilde{C_{1}}$ is Hurwitz, $e_{\overline{x_{1}}}$ will converge exponentially to zero.

The existence conditions of the observer in the equation (19), are listed in [8] and [9] as:

- $\operatorname{rank}(C D)=\operatorname{rank}(D)=l$

- $\operatorname{rank}(C B)=\operatorname{rank}(B)=p$

- $\left(\tilde{A}_{1}, \tilde{C}_{1}\right)$ is observable (detectable)

\subsubsection{Fault Detection and Localisation}

In order to detect faults, residual signals that indicate the presence of abnormal situations must be generated and evaluated. 
The residuals generation based on observer is synthesized from the discrepancy between the system outputs and their estimation.

From (16-a) and (16-b), the estimation of the original system states given by (8) is obtained as:

$$
\begin{aligned}
& \hat{x}_{1}(t)=T \hat{\bar{x}}_{1}(t)=T w(t) \\
& \hat{x}_{2}(t)=T \hat{\bar{x}}_{2}(t)=T\left(U_{1} y(t)-U_{1} C N w(t)\right) \\
& \hat{x}_{3}(t)=T \hat{\bar{x}}_{3}(t)=T\left(U_{2} y(t)-U_{2} C N w(t)\right)
\end{aligned}
$$

Therefore, from (21), the residual vector is given by:

$$
r(t)=y(t)-\hat{y}(t)=C(x(t)-\hat{x}(t))
$$

The distinction between the faults and the other disturbances effects on the residual signal is done by the definition of a threshold $r_{t h}$ so that:

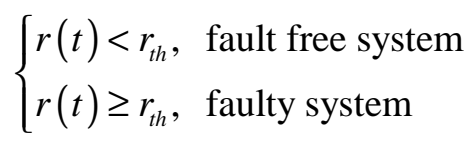

The UIO allows a robust fault generation by decoupling the disturbances effect from the faults effects so that the residues are sensitive just to faults and not to unknown inputs. This robustness has the consequence of choosing $r_{t h} \sqcup 0$.

The residues are influenced by all the faults that can affect the system since the different system states are related. To encounter this problem and guarantee the fault localisation, a bank of UIO is used. This method authorizes to decouple the effect of a fault from another one.

In this work, we refer to a Dedicated Observer Schema DOS of UIO to isolate the faulty actuator as presented in the figure 2 :

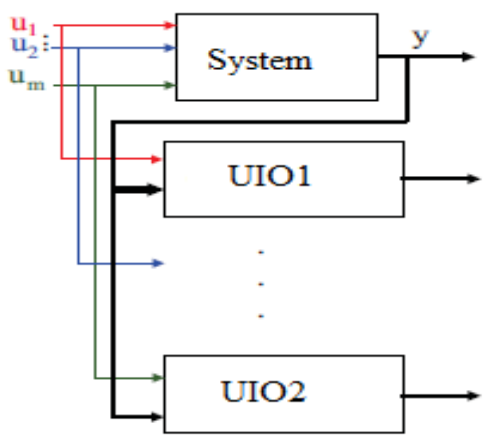

Figure 2. DOS for faults localisation. 


\subsubsection{Fault Estimation}

A fault estimation $\hat{f}$ of $f$ can be found through an algebraic management of the differential equations matching to the transformed state vector $\bar{x}_{3}$ in (10) and through the reduced-order observer (19) and the equation (21):

$$
\begin{aligned}
\hat{f}(t) & =\dot{\bar{x}}_{3}(t)-\left[\begin{array}{lll}
\bar{A}_{31} & \bar{A}_{32} & \bar{A}_{33}
\end{array}\right] \hat{\bar{x}}(t)-\bar{B}_{3} u(t) \\
& =U_{2} \dot{y}(t)+G_{1} w(t)+G_{2} y(t)+G_{8} u(t)
\end{aligned}
$$

where $\hat{f} \in \mathfrak{R}^{p}$ and the terms $G_{1}, G_{2}$ and $G_{2}$ are given by:

$$
\begin{aligned}
& G_{1}=U_{2} C N\left(L U_{3} C N-\bar{A}_{11}+\bar{A}_{12} U_{1} C N+\bar{A}_{13} U_{2} C N\right)-\bar{A}_{31}+\bar{A}_{32} U_{1} C N+\bar{A}_{33} U_{2} C N \\
& G_{2}=U_{2} C N\left(-L U_{3}-\bar{A}_{12} U_{1}-\bar{A}_{13} U_{2}\right)-\bar{A}_{32} U_{1}-\bar{A}_{33} U_{2} \\
& G_{3}=-U_{2} C N \bar{B}_{1}-\bar{B}_{3}
\end{aligned}
$$

The fault estimation error is given by:

$$
\begin{aligned}
& e_{f}(t)=f(t)-\hat{f}(t) \\
& =U_{2} C N e_{\overline{\bar{x}}_{1}}(t)+\left(\bar{A}_{31}-\bar{A}_{32} U_{1} C N-\bar{A}_{33} U_{2} C N\right) e_{\bar{x}_{1}}(t)
\end{aligned}
$$

The error $e_{f}$ exponentially converges to zero since $e_{\bar{x}_{1}}$ converges to zero.

By the same way it is also possible to estimate the unknown input $d(t)$, however, we are just interested by the fault estimate which will be exploited in the FTC task.

\subsection{Fault accommodation}

While developing an active fault tolerant control approach, in some works it is assumed that the fault detection, isolation and identification are perfect. However, in practise it is not possible to find exact and immediate information about the fault. Therefore, in this section, the fault accommodation procedure will use the fault estimation to recalculate on line the control parameters $K_{n}$ and $G_{n}$ given by equation (5) in the nominal case to obtain $K_{r}$ and $G_{r}$ for the reconfigurationlaccommodation mode.

The estimation of the loss of control factor efficiency $\hat{\Gamma}$ is extracted from the fault estimation $\hat{f}$ and from the relation (7) by:

$\hat{\Gamma}(t)=\operatorname{diag}\left(\hat{\gamma}_{1}(t), \hat{\gamma}_{2}(t), \cdots, \hat{\gamma}_{p}(t)\right)=\hat{f}(t) u^{+}(t)$

The control gains calculated on line after the fault detection are, then, uttered as:

$$
\left\{\begin{array}{l}
K_{r}(t)=\left(B\left(I_{p}-\hat{\Gamma}(t)\right)\right)^{+}(A-M) \\
G_{r}(t)=\left(B\left(I_{p}-\hat{\Gamma}(t)\right)\right)^{+} N
\end{array}\right.
$$


The accommodation control applied from a reconfiguration instant $t_{r}$ is then expressed by:

$u(t)=G_{r}(t) e(t)-K_{r}(t) \hat{x}(t), \quad t \in\left[t_{r}, t_{m i s}\right]$

Before the convergence of $\hat{\Gamma}$ to the true value of the factor efficiency's loss, the discrepancy between the true and the estimated value can be important and influences badly the quality of the accommodating control law given in (26).

For this reasons, we propose a decision function to activate the reconfiguration mechanism at an adequate time for which the estimation error is relatively small. The activation function can be represented as:

$$
\begin{cases}e_{f}(t)>\delta_{t h}, & \text { do not accommodate } \\ e_{f}(t) \leq \delta_{t h}, & \text { apply accommodating control }\end{cases}
$$

The activation threshold $\delta_{t h}$ must be significantly chosen so that the estimation error value is acceptable, in one side. In the other side, the choice of $\delta_{t h}$ should not cause an unacceptable reconfiguration delay.

\section{ILLUSTRATIVE EXAMPLE}

The previous analysis is applied on a "Vertical Take-off and Landing" aircraft model with a dynamic linearization at an airspeed evaluated by $250.02 \mathrm{Km} / \mathrm{h} \mathrm{[10]}$.

The aircraft model is represented under a state space formulation that is similar to equation (8), where its parameters are as following:

$$
\begin{aligned}
A & =\left[\begin{array}{cccc}
-0.0366 & 0.0271 & 0.0188 & -0.4555 \\
0.0482 & -1.01 & 0.0024 & -4.0208 \\
0.1002 & 0.3681 & -0.7070 & 1.42 \\
0 & 0 & 1 & 0
\end{array}\right] \\
B & =\left[\begin{array}{cc}
0.4422 & 0.1716 \\
3.5446 & -7.5922 \\
-5.52 & 4.49 \\
0 & 0
\end{array}\right], C=\left[\begin{array}{cccc}
1 & 0 & 0 & 0 \\
0 & 1 & 0 & 0 \\
0 & 0 & 1 & 0 \\
0 & 1 & 1 & 1
\end{array}\right]
\end{aligned}
$$

The state variables $x=\left(\begin{array}{llll}x_{1} & x_{2} & x_{3} & x_{4}\end{array}\right)^{\mathrm{T}}$ represent respectively, the horizontal velocity the vertical velocity, the pitch rate and the pitch angle. The control input $u_{1}$ is the collective pitch control and $u_{1}$ is the longitudinal cyclic pitch angle.

The control objective is to make the closed loop system behavior follow the stable reference model given by (27) within a time $t_{\text {mis }}=7 \mathrm{sec}$ : 
International Journal of Instrumentation and Control Systems (IJICS) Vol.2, No.3, July 2012

$\dot{x}(t)=\left[\begin{array}{cccc}-0.8278 & 0 & 0 & 0 \\ 0 & -1.0105+j 1.5147 & 0 & 0 \\ 0 & 0 & -1.0105-j 1.5147 & 0 \\ 0 & 0 & 0 & -2.5513\end{array}\right] x(t)+B\left[\begin{array}{c}0.5 \\ 1\end{array}\right]$

The simulations are done for initial conditions $x(0)=\left(\begin{array}{llll}0 & 0 & 0 & 0\end{array}\right)^{\mathrm{T}}$. The control law for the fault free mode is given by:

$u(t)=\left[\begin{array}{c}0.5 \\ 1\end{array}\right]-\left[\begin{array}{cccc}7.5417 & 0.5117 & -1.8532 & -7.5164 \\ 4.4390 & 0.3060 & -0.9332 & -3.7883\end{array}\right] x(t)$

Figure 3 represents the nominal system's behaviour; without faults and disturbances. The nominal outputs are stable and follow the reference input.
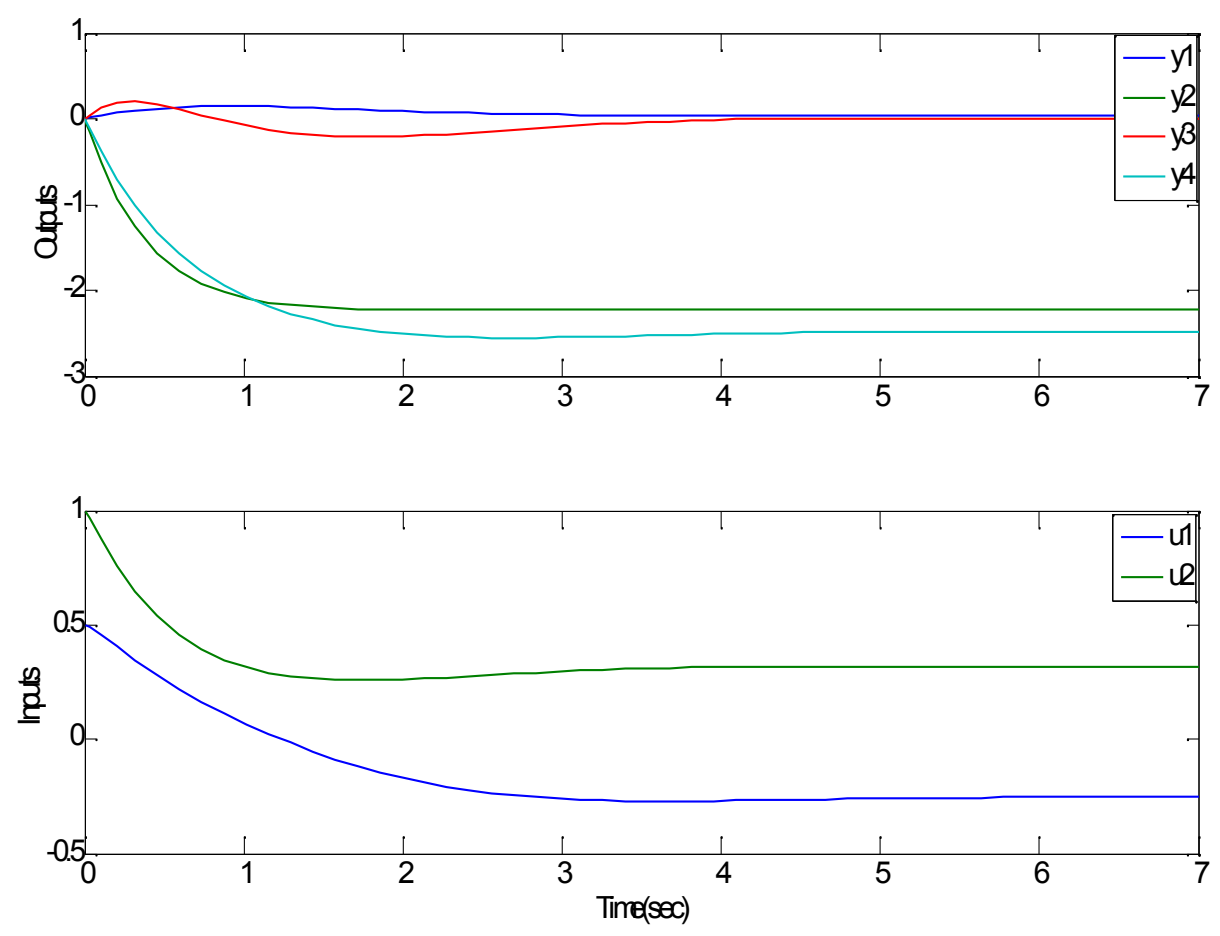

Figure 3. System behaviour in nominal mode.

The system is subject to an unknown input and fault in the second actuator, described by the following equations:

$D d(t)=\left(\begin{array}{llll}0 & 0 & 1 & 0\end{array}\right)^{\mathrm{T}} d(t), d(t)=\left\{\begin{array}{l}0, t<1 \mathrm{sec} \\ {\left[\begin{array}{llll}0 & 0.5 & 0.2 & 0\end{array}\right] x(t), t \geq 1 \mathrm{sec}}\end{array}\right.$ 
$\gamma_{2}=\left\{\begin{array}{l}0 \%, t<2 \mathrm{sec} \\ 70 \%, t \geq 2 \mathrm{sec}\end{array}\right.$

The system outputs and inputs in the faulty mode are represented in the figure 4 . The effects of the disturbance and the fault are quite clear on the input signals.
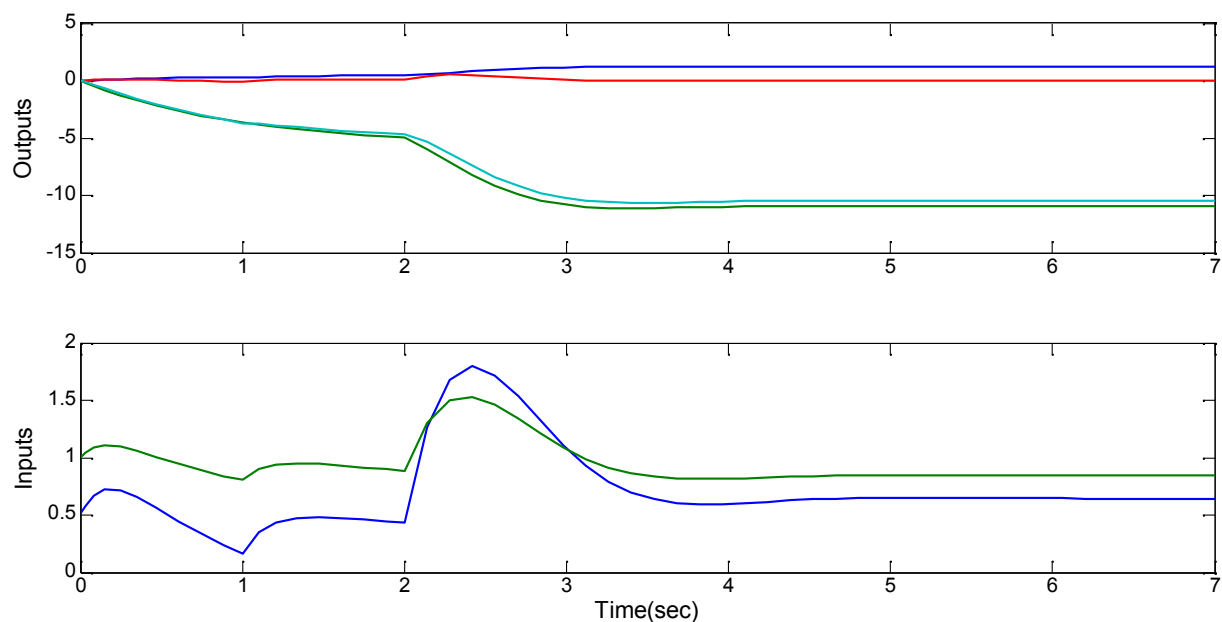

Figure 4. System behavior in presence of fault and disturbance.

The fault detection and isolation is done by a bank of UIO constituted by two UIO, in a DOS structure, such that UIO1 for the first actuator and UIO2 for the second actuator. The residues supplying from UIO1 and UIO2 are illustrated on the figure 5. Besides, it is clear that the residues of the UIO1 are insensitive to the fault of the second actuator. However, the residues of the UIO2 are sensitive to the fault and not to the unknown input. This shows the robustness of the residual generation by UIO.
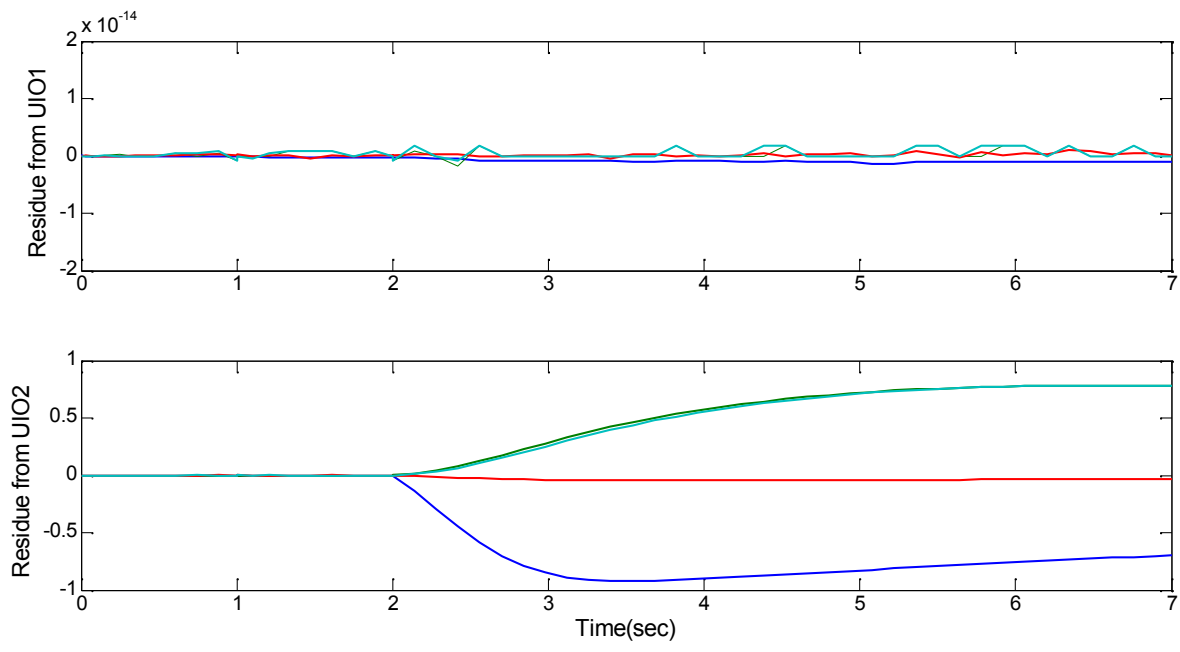

Figure 5. Residual signals from UIO1 and UIO2. 
International Journal of Instrumentation and Control Systems (IJICS) Vol.2, No.3, July 2012

Figure 6 and figure 7 show respectively the true and the estimated value of the loss of efficiency and the error estimation. The estimated value converges to the true one within about 3 seconds.

Figure 6. Real and estimated value of $\gamma_{2}$.
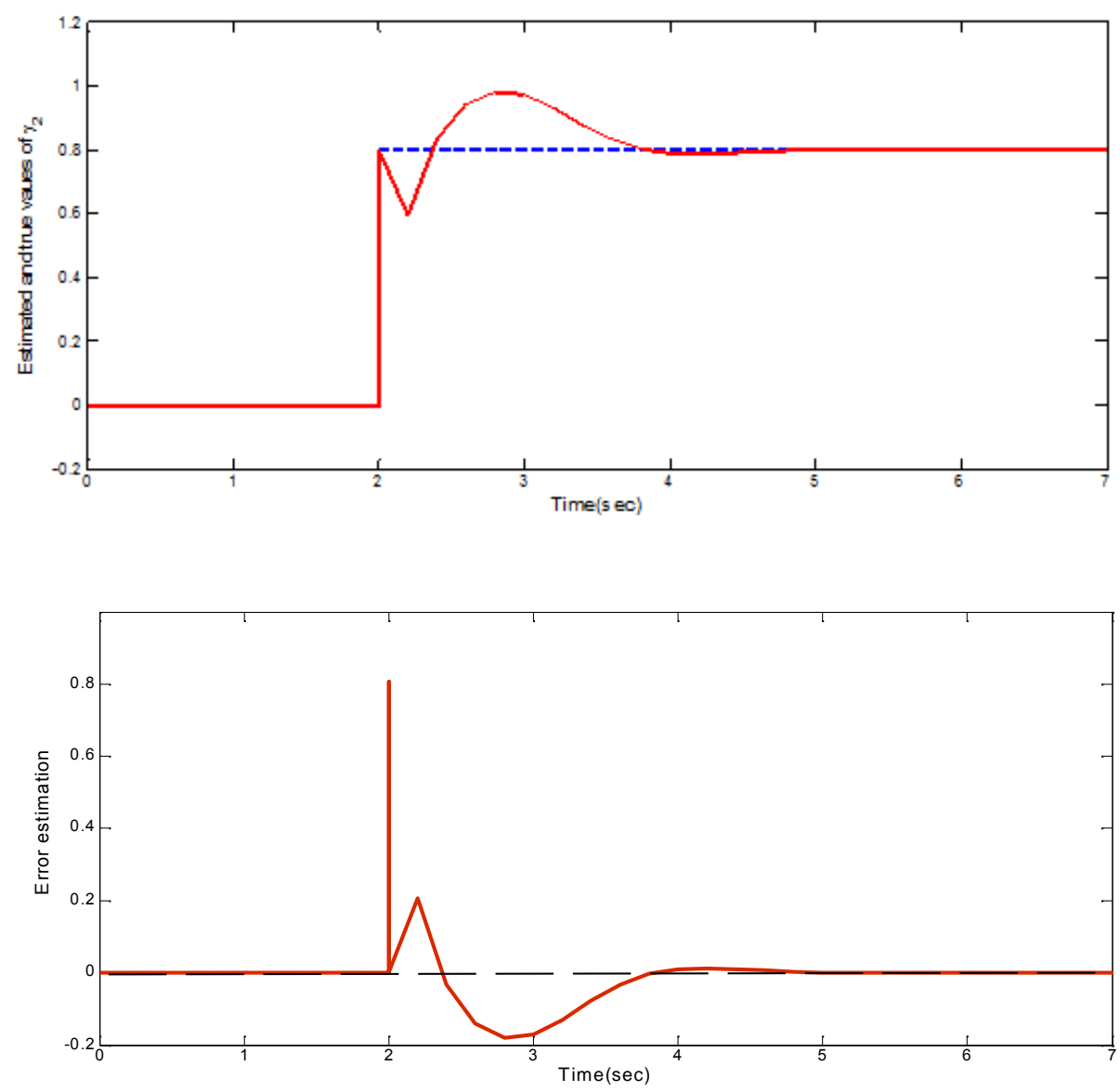

Figure 7. Evolution of the error estimation.

By fixing a threshold of the activation function $\delta_{t h}=10^{-3}$, the instant of applying the accommodating control is given by $t_{r}=4.9 \mathrm{sec}$. The system response after the fault accommodation is illustrated by figure 8 . The fault effect is compensated and the outputs after accommodation, in the degraded mode, join the nominal outputs. 

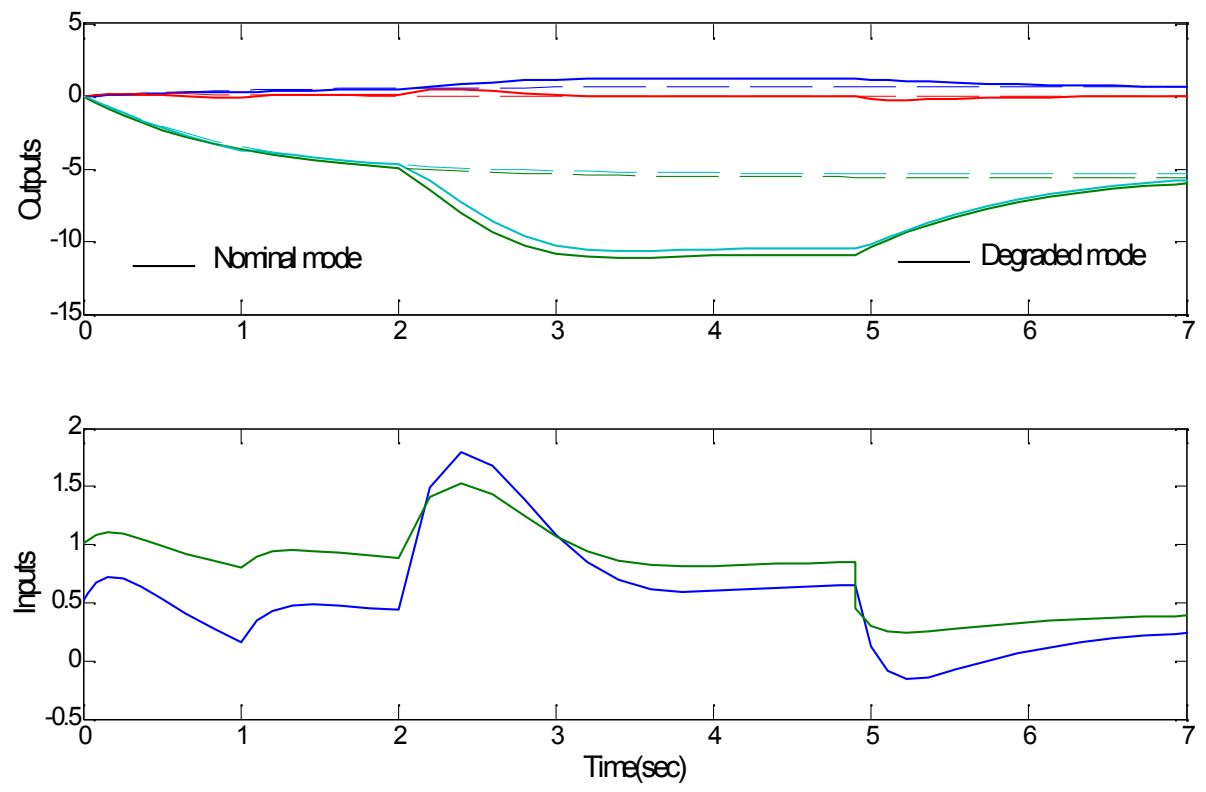

Figure 8. System response with fault accommodation.

\section{CONCLUSIONS}

This paper presented an integrated design of actuator fault detection, diagnosis and accommodation. The Fault Detection and Diagnosis is based on an unknown input observer allowing a robust diagnosis, fault isolation and estimation. The fault tolerant control based on the Pseudo-inverse method uses the information from the FDD module and applies an accommodating control in a chosen instant. The optimal choice of this reconfiguration instant has been studied in many works $[11,12]$.

Simulation results, on an aircraft model, show the supply of the theoretical developments in the problems of links between the FDD and the FTC modules. Future works will be concerned by a temporal study of the integrated architecture.

\section{REFERENCES}

1] Zhang, Y.M. \& Jiang, J. , Bibliographical review on reconfigurable fault-tolerant control systems, Proceeding of the SAFEPROCESS, 5th Symposium on Detection and Safety for Technical Processes, IFAC, 265-276, 2003.

[2] Isermann, R. Model-based fault-detection and diagnosis--status and applications, Annual Reviews in control, Volume $29 \mathrm{n}^{\circ} 1$, Elsevier, 2005.

[3] M. Mahmoud, J. Jiang, Y.M. Zhang, Fault Detection and isolation using unkown input observers with structured residual generation , International Journal of Instrumentation and Control Systems (IJICS) Vol.2, No.2, April 2012.

[4] Zhang, X., Polycarpou, MM \& Parisini, T., Integrated design of fault diagnosis and accommodation schemes for a class of nonlinear systems, Decision and Control, Proceedings of the 40th IEEE Conference, Volume 2, 2001.

[5] Zhang, Y. and Jiang, J., Design of integrated fault detection, diagnosis and reconfigurable control systems, Decision and Control, Proceedings of the 38th IEEE Conference, Volume 4, 1999. 
[6] Staroswiecki, M. \& Cazaurang, F., Fault recovery by nominal trajectory tracking, American Control Conference, Volume $32 \mathrm{n}^{\circ} 2,2008$.

[7] Maki, M. \& Hagino, K., A stability guaranteed active fault tolerant control system against actuator failures, International Journal of Robust and Nonlinear Control, Volume 14, 2004.

[8] Sotomayor, O.A.Z. \& Odloak, D., Observer-based fault diagnosis in chemical plants, Chemical Engineering Journal, Volume $112 \mathrm{n}^{\circ}$ 1, Elsevier, 2005.

[9] Park, T.G. \& Lee, K.S., Process fault isolation for linear systems with unknown inputs, Control Theory and Applications, IEE Proceedings, Volume 151 n $^{\circ}$, IET, 2004.

[10] Narendra, K., and Tripathi, S.S. 'Identification and optimization of aircraft dynamics'. DTIC Document., 1973.

[11] Hamdaoui, R., and Abdelkrim, M.N.: 'Temporal analysis of actuator fault recovery based on optimal control', International Review of Automatic Control IREACO., vol4, n² 2011.

[12] Hamdaoui, R., and Abdelkrim, M.N.: 'Conditions on diagnosis and accommodation delays for actuator fault recoverability', 8th International Multi-Conference on Systems, Signals \& Devices., IEEE, 2011. 